\title{
Vom Umgang mit Überkomplexität
}

\section{Professionelle Urteilsbildung im Rahmen der Alterseinschätzung junger Geflüchteter}

Die Alterseinschätzung junger Geflüchteter wird im öffentlichen Raum deshalb so kontrovers diskutiert, weil sie überaus komplex ist. Fehlende Ausweispapiere, strategische Kommunikation und vage formulierte, rechtliche Vorgaben lenken den Blick in diesem Beitrag auf die Praxis der qualifizierten Inaugenscheinnahme: Wie werden Fallwissen und Urteilsvermögen im Hinblick auf das Alter junger Geflüchteter hergestellt?

I m Jahr 2016 wurden knapp 45.000 Minderjährige nach unbegleiteter Einreise durch die Jugendämter in Deutschland in Obhut genommen (Destatis 2020). Unbegleitete minderjährige Ausländer (UMA) ${ }^{1}$ machten damit mehr als die Hälfte aller in dem Jahr durchgeführten Inobhutnahmen aus (ebd.). Anders als in europäischen Nachbarländern, wie z. B. in Frankreich, sind die Zahlen in Deutschland seitdem kontinuierlich rückläufig: 2019 wurden lediglich noch 8647 unbegleitete Einreisen registriert, also etwa ein Fünftel im Vergleich zu 2016 (ebd.). ${ }^{2}$

Im Zuge verschiedener Gesetzesnovellierungen sowie der Weiterentwicklung von Strukturen und Verfahren wurde seit 2014 auf die starke Zuwanderung reagiert (Brinks et al. 2016; Tangermann und Hoffmeyer-Zlotnik 2018). So wurden zum Beispiel mit dem Gesetz zur

Hannes Käckmeister 1,2
'Université de Strasbourg, Straßburg, Frankreich
2Pädagogische Hochschule Freiburg, Freiburg, Deutschland
*1990; Deutsch-französische Politikwissenschaften, Doktorand
im binationalen Promotionsverfahren an der PH Freiburg und der
Universität Straßburg.
Hkaeckmeister@unistra.fr

Zusammenfassung Der Beitrag zeigt, worin die Komplexität der Alterseinschätzung begründet liegt und wie Sozialarbeitende in der Praxis der qualifizierten Inaugenscheinnahme damit umgehen. Grundlage des Beitrags ist eine soziologische Studie zu den Verfahren der Alterseinschätzung in Deutschland und Frankreich, die im Rahmen einer Doktorarbeit durchgeführt wurde, die voraussichtlich im zweiten Halbjahr 2021 veröffentlicht wird. Das methodologische Vorgehen beruht auf ethnographischen Beobachtungen und Interviews, die zwischen 2017 bis 2019 in einem Jugendamt durchgeführt wurden.

Schlüsselwörter Qualifizierte Inaugenscheinnahme, Alterskategorien, Komplexität, Unbegleitete minderjährige Ausländer
Verbesserung der Unterbringung, Versorgung und Betreuung ausländischer Kinder und Jugendlicher im November 2015 ein bundesweiter Verteilmechanismus und ein behördliches Verfahren zur Alterseinschätzung eingeführt (Bundesgesundheitsblatt 2015). Die Ziele: Verfahren harmonisieren und Zuständigkeiten klären. In der öffentlichen Debatte lässt sich eine Verschiebung des medialen und gesellschaftlichen Diskurses von Unterbringungs- und Integrationsfragen hin zu einer problemzentrierten Perspektive auf ,UM` beobachten. Die Alterseinschätzung wird dabei wiederkehrend besonders kontrovers diskutiert (Tangermann und Hoffmeyer-Zlotnik 2018, S. 24).

\section{Das Streben nach Evidenz auf der Basis von Misstrauen und Unsicherheit}

Die Alterseinschätzung erfolgt seit 2015 in einem dreistufigen Verfahren ( $\mathbb{4} 42 \mathrm{f}$ SGB VIII). Liegen gültige Ausweispapiere vor, dann ist das darin angegebene Alter maßgeblich. Hilfsweise wird von dem zuständigen Jugendamt eine qualifizierte Inaugenscheinnahme durchgeführt, in der die mündlichen Angaben der jungen Geflüchteten geprüft und deren äußeres Erscheinungsbild durch Sozialarbeitende in Augenschein genommen wird. In „Zweifelsfällen“ sollen ärztliche Untersuchungen eingeleitet werden (z. B. Begutachtungen des Gebisses, der Zahnreife und/oder des Entwicklungsstands sowie Röntgendiagnostik zur Analyse des Schlüsselbeins, der Handwurzelknochen oder der Zähne). Bestehen nach ärztlichen Untersuchungen Zweifel fort, dann ist von Minderjährigkeit auszugehen. ${ }^{3}$ Das mehrstufige Verfahren ist eng mit Misstrauen und Unsicherheit verbunden und offenbart in der Praxis die Komplexität der Alterseinschätzung.

Erstens zeigen die Erkenntnisse aus der Forschungsarbeit, dass Ausweis- und Passdokumente nur in Ausnahmefällen vorliegen. Das ist im Kontext Flucht nicht ju- 


\section{Extrablick: Urteilsbildung in der Sozialen Arbeit}

gendspezifisch und kann sehr unterschiedliche Gründe haben: In vielen Herkunftsländern gibt es kein engmaschiges, behördliches Pass- und Meldewesen (Europarat 2019, S. 13; UNICEF 2019), was u. a. an Kriegen und bewaffneten Konflikten liegen kann (Parusel 2015, S. 22). Darüber hinaus gibt es Fälle, in denen Dokumente auf der Flucht abhandengekommen sind (verloren, von Schleusern abgenommen etc.) oder schlichtweg nicht vorgezeigt werden. Diese unterschiedlichen Szenarien haben die gemeinsame Konsequenz, dass amtlich ausgestellte und zertifizierte Dokumente nur selten als Entscheidungsgrundlage für die Alterseinschätzung zur Verfügung stehen.

Zweitens gibt es bis dato keine Methode - ob medizinisch oder nicht-medizinisch - mit der sich das Alter einer Person unstrittig und hinreichend valide bestimmen lässt (Bundesärztekammer 2016; Bundesarbeitsgemeinschaft Landesjugendämter 2017; European Asylum Support Office 2018): Während die qualifizierte Inaugenscheinnahme subjektiv ist und lediglich Einschätzungen ermöglicht, liefert medizinische Altersdiagnostik zwar objektive und wissenschaftliche Validität suggerierende Resultate. In einem Graubereich von ein bis zwei Jahren handelt es sich jedoch ebenfalls nur um Näherungswerte (Bundesarbeitsgemeinschaft Landesjugendämter 2017, S. 37).

Die qualifizierte Inaugenscheinnahme ist vor diesem Hintergrund mit Unsicherheit verbunden und Unsicherheit schafft Komplexität. Vertrauen in die Aussagen der jungen Geflüchteten würde Unsicherheit überbrücken und Komplexität reduzieren (Luhmann 2014) - jedoch beruht die qualifizierte Inaugenscheinnahme auf der Prämisse der strategischen Kommunikation, welche als „Interessenhandeln“ (Habermas 1982, S. 383) nicht in Vertrauen, sondern in Zweifeln und Misstrauensunterstellungen begründet liegt. Mit anderen Worten werden die Altersangaben der jungen Geflüchteten nicht als selbstverständlich glaubhaft betrachtet, sondern deren Richtigkeit hinterfragt und eine Überprüfung für notwendig erachtet. Warum das so ist, soll - als dritte Herausforderung formuliert - im Folgenden geklärt werden. Die jungen Geflüchteten sollen vertrauenswürdig erscheinen, damit auch ihre Altersangaben als glaubwürdig eingeschätzt werden. Von ihnen wird erwartet, dass sie sich öffnen und den Sozialarbeitenden Einblicke in ihr Leben vor, während und nach der Flucht gewähren - und dass sie diese Einblicke während des Interviews ausdrücken und explizieren können. Damit wird ein interpersonales und kategoriales (im Hinblick auf die Sozialarbeitenden als Repräsentanten einer staatlichen Institution) Vertrauen vorausgesetzt. Aufgrund des institutionellen Gesprächscharakters (folgenreiche Entscheidung, asym- metrische Gesprächskonstellation, kurze Gesprächsdauer) und der individuellen Fluchterfahrungen der Personen lassen sich Misstrauen und strategische Kommunikation jedoch als „notwendige Überlebensstrategie[n]“ bewerten (Scherr 2014, S. 314), die den Verlauf der qualifizierten Inaugenscheinnahme beeinflussen. Während die jungen Geflüchteten also vertrauenswürdig erscheinen sollen, wird von den Sozialarbeitenden erwartet, vertrauensfähig zu sein, denn die Selbstauskunft der Betroffenen darf nur in begründeten Fällen angezweifelt werden („Primat der Selbstauskunft“). Diese Vertrauensfähigkeit ist jedoch ebenfalls eingeschränkt, denn die Altersangaben werden potenziell als Ausdruck strategischer Kommunikation betrachtet. So berichten die Sozialarbeitenden von ihrem Eindruck, permanent getäuscht zu werden: Auch Volljährige würden versuchen, sich minderjährig zu machen, um Zugang zu Leistungen der Jugendhilfe und bessere Integrationsperspektiven zu erhalten. Sie entwickeln, so lässt sich dieser Eindruck auch formulieren, kreative Taktiken, um sich den Anforderungen der aktuellen Rechtslage und den folgenreichen Auswirkungen der Altersgrenze anzupassen. Strategische Kommunikation, Misstrauen und Unsicherheit führen zu Überkomplexität: Es müssen Einschätzungen vorgenommen und Entscheidungen getroffen werden, die in vielen Situationen in mangelndem Urteilsvermögen begründet liegen und die mit Fallstricken und Dilemmata verbunden sind. Und das Mandat dafür liegt in erster Linie bei der Sozialen Arbeit. Wie gehen aber die Sozialarbeitenden in der Praxis damit um?

\section{Körperlichkeit als Hilfskonstruktion für die Urteilsbildung}

Qualifizierte Inaugenscheinnahmen werden durch zwei erfahrene sozialpädagogische Fachkräfte, ggf. mit Hilfe einer psychologischen Fachkraft oder einer Verwaltungsfachkfraft des Jugendamts (Bundesarbeitsgemeinschaft Landesjugendämter 2017, S. 57) und im Beisein einer sprachmittelnden Person durchgeführt. In ihren Einschätzungen sollen sie sich an verschiedenen Kriterien orientieren, die als vergleichbare Maßstäbe für Minderjährigkeit, bzw. Volljährigkeit politisch und gesellschaftlich anerkannt sind: äußeres Erscheinungsbild, Entwicklungsstand, Auftreten und mündliche Aussagen. Die diesem Beitrag zugrunde liegende Studie zeigt, dass die Fachkräfte aufgrund fehlender Benennung und Differenzierung dieser Kriterien im deutschen Kinder- und Jugendhilferecht alternativen Orientierungspunkten folgen. $\mathrm{Zu}$ diesen zählen die Handlungsempfehlungen der Bundesarbeitsgemeinschaft der Landesjugendämter $(2014,2017)$ sowie Modelle anderer Jugendämter. Qualifizierte Inaugenscheinnahmen sollen schrift- 
lich dokumentiert werden (s. dazu z. B. das Urteil vom VGH München 2017, B.v. 05.04.2017-12 BV 17.185, Rn. 32). In dem Jugendamt, in dem die Forschung zwischen 2017 und 2019 durchgeführt wurde, ist die Falldokumentation in einen biographischen Anamneseteil und einen Entscheidungsteil untergliedert. In letzterem wird das Kriterium des äußeren Erscheinungsbilds in verschiedene Merkmalsausprägungen unterteilt und dadurch operationalisiert. Zu ihnen zählen „ausgeprägte Stirnfalte“, „starke Körperbehaarung “, „ausgeprägte Halsfalten“, „,ergraute Haare“, „Bartwuchs“ und „postpubertärer Körperbau“.

Diese Merkmalszuschreibungen dienen dazu, die subjektiven Einschätzungen an vergleichbaren, vermeintlich objektiven Indikatoren festzumachen, um Entscheidungen herbeiführen und rechtfertigen zu können. Es lässt sich jedoch kritisch anführen, dass es sich um kommunikativ - also in der Gesprächsinteraktion - vermittelte Hilfskonstruktionen handelt, in denen sich Männlichkeitsbilder sowie ethnozentrierte und stereotype Vorstellungen von Kindheit, Jugend und Erwachsenwerden manifestieren. Junge Geflüchtete aus mehrheitlich afrikanischen (z. B. Guinea, Elfenbeinküste oder Eritrea) und asiatischen (z. B. Afghanistan und Syrien) Ländern werden anhand äußerer Erscheinungsmerkmale einer Alterskategorie (Minderjährigkeit oder Volljährigkeit) zugeordnet. Die formale Anforderung an die qualifizierte Inaugenscheinnahme - als Praxis der Kategorisierung - trifft auf die rechtlich verankerten Vorgaben und den von den Sozialarbeitenden formulierten Anspruch, vorsichtig mit Stereotypisierungen und Generalisierungen umzugehen und den Gleichbehandlungsgrundsatz zu respektieren.

Es gibt daher widersprüchliche Anforderungen an die fachliche Urteilsbildung (vgl. Carayon et al. 2018, S. 40), die in der Anwendungspraxis u. a. durch Heuristiken (Analogien und Kategorisierungen) - d. h. durch kognitive Prozesse, in denen komplexe Situationen vereinfacht werden - aufgelöst werden. Es werden bspw. Fälle mit vorangegangenen Fällen verglichen, die aus Sicht der Sozialarbeitenden ähnlich waren. In diesen Fällen werden kulturalisierende und stereotype Vorstellungen darüber entwickelt, wie Minderjährige in bestimmten Ländern aussehen (Carayon et al. 2020). Dabei sind die Sozialarbeitenden selbst nie vor Ort gewesen und sie bekommen nur in seltenen Fällen - etwa nach Gerichtsentscheidungen - Rückmeldungen zu der „Richtigkeit“ ihrer Einschätzung. Ihr Erfahrungswissen ist folglich in Annahmen und Analogien vorangegangener Fälle begründet, was dazu führt, dass sich stereotype Vorstellungen in der Anwendungspraxis reproduzieren und als Routinen festsetzen - auch dann, wenn die Sozialarbei- tenden das eigentlich gar nicht wollen und für sich den Anspruch vertreten, „gute“ und „gerechte“ Entscheidungen zu treffen. Dass bei Personen im Grenzbereich zwischen Voll- und Minderjährigkeit daher nicht allein das Kriterium des „äußeren Erscheinungsbildes“ oder des „Entwicklungsstandes“ Aufschluss über deren Alter geben kann, betont u. a. das VGH München in seinem Beschluss vom 05. April 2017 (2017, 12 BV 17.185, Rn. 50). Von den qualifizierten Fachkräften der Jugendhilfe wird folglich neben Aussehen und Verhalten auch die Plausibilität der Aussagen der jungen Geflüchteten in die Bewertung einbezogen.

\section{Der Umgang mit Widersprüchen}

Während der qualifizierten Inaugenscheinnahme sollen die jungen Geflüchteten Angaben u. a. zu dem Alter ihrer Eltern und Geschwister, den Daten ihrer Einschulung und Beendigung der Schule, den Arbeits- und Berufsfeldern der Eltern sowie zu Fluchtwegen und -zeiten machen. Es geht darum, Indizien zu sammeln, die Rückschlüsse auf das Alter der Personen zulassen. Dabei können angenommene Widersprüche in den Erzählungen mögliche Zweifel an der Minderjährigkeit verstärken. Die Einschätzung des Alters auf der Grundlage von Fluchtbiographien und Aussagen während der qualifizierten Inaugenscheinnahme führt im Hinblick auf die Urteilsbildung jedoch zu einem weiteren Dilemma, denn welche Rückschlüsse lassen als widersprüchlich empfundene Angaben auf Minderjährigkeit und Volljährigkeit überhaupt zu?

Psychologische Studien zum Beispiel zeigen, dass traumatisierende Erlebnisse im Kontext von Flucht zu kognitiven Verdrängungsprozessen und verzerrten Erinnerungen führen können (Montagut 2016). Mit anderen Worten: Sich an Zeitverläufe, Daten und Namen nicht erinnern, bzw. diese im Rahmen der qualifizierten Inaugenscheinnahme nicht auf den Punkt abrufen und explizieren zu können, muss nicht auf trügerische Absichten hinweisen, sondern kann im Gegenteil auch ein Zeichen für Hilfebedarf sein. Angenommene Unwahrheit kann darüber hinaus auch Ausdruck von Jugendlichkeit sein. $\mathrm{Zu}$ diesem Urteil kommt auch das Verwaltungsgericht Hannover: „Es ist gerichtsbekannt, dass auch Jugendliche häufig die Unwahrheit sagen. Gerade die Offensichtlichkeit der Widersprüche in den Angaben des Antragstellers lassen zudem bei vorläufiger Würdigung mindestens genauso gut den Schluss zu, dass der Antragsteller noch nicht die Reife eines Erwachsenen erreicht hat." (VG Hannover 2016, B.v. 11.11.2016-3 B 5176/16, Rn. 19). Folglich rechtfertigen Widersprüche an sich nicht die Schlussfolgerung einer falschen Altersangabe. 


\section{Extrablick: Urteilsbildung in der Sozialen Arbeit}

Hinzu kommt, dass Alter und Geburtstage in anderen Ländern und Kulturkreisen nicht denselben Stellenwert haben wie in Europa (VGH München 2017, B.v. 05.04.2017-12 BV 17.185, Rn. 30), was wiederum dazu führt, dass einige junge Geflüchtete konkrete Altersangaben schlichtweg nicht machen können. Es stellt sich daher die Frage, wie verfahren wird, wenn die Einschätzungen auf der Grundlage des äußeren Erscheinungsbildes und der mündlichen Aussagen keine konkreten Ergebnisse zulassen.

\section{Vom Vier-Augen-Prinzip zur Sechs-Augen-Realität}

Der sogenannte „Zweifelsfall“ ist in Deutschland ein rechtlich relevanter Begriff, denn er markiert den Übergang von der qualifizierten Inaugenscheinnahme zu medizinischen Untersuchungen. Ein „Zweifelsfall“ liegt dann vor, wenn im Rahmen der qualifizierten Inaugenscheinnahme nicht mit Sicherheit ausgeschlossen werden kann, dass ein fachärztliches Gutachten zu dem Ergebnis kommen wird, der Betroffene sei noch minderjährig (VGH München 2016, v. 18.08.2016, 12 CE 16.1570, Rn. 14). Mit anderen Worten: Wenn junge Geflüchtete nicht offensichtlich volljährig sind, dann muss eine medizinische Untersuchung eingeleitet werden, „um Fehlbeurteilungen zu Lasten von Minderjährigen zu vermeiden.“ (VG München 2016, v. 07.12.2016, M 18 K 16.4361, Rn. 21).

In der Anwendungspraxis führen „Zweifelsfälle“ zu einem Ermessensspielraum für die Sozialarbeitenden, denn „Zweifelsfälle“ lassen sich nicht an eindeutigen und wissenschaftlichen Indikatoren bemessen; vielmehr handelt es sich um situative und individuelle Konstruktionen. Hierin liegt einer der Gründe dafür, dass es erhebliche Disparitäten im Hinblick auf die Verfahren und Methoden innerhalb Deutschlands gibt (Deutsche Bundesregierung 2020, S. 69; Deutscher Caritasverband e. V. 2018, S. 3). Beispielsweise variiert der Rückgriff auf medizinische Alterseinschätzungen stark: Während u. a. in Bremen, Hamburg und Sachsen-Anhalt eine regelmäßige Praxis medizinischer Alterseinschätzungsverfahren besteht, werden diese in Sachsen und Rheinland-Pfalz kaum angewendet (Karpenstein und Rohleder 2021, S. 26). Zum Teil werden Alterseinschätzungen auch durch Ausländerbehörden durchgeführt, die ihrerseits Zweifel am Alter der Person äußern und medizinische Alterseinschätzungen veranlassen können (Karpenstein und Nordheim 2020, S. 21). In diesen Fällen ist dann die Gesetzesgrundlage das Aufenthalts- und nicht das Kinder- und Jugendhilferecht, wodurch die Primärzuständigkeit der Kinder- und Jugendhilfe in Frage gestellt, wenn nicht gar unterlaufen wird. Für die Soziale Arbeit ergibt sich aus diesen unterschiedlichen Macht- und Kompe- tenzverteilungen eine doppelte Anforderung: Anpassung an politische Interessen und Entscheidungen auf Ebene der Länder und Kommunen einerseits und fachliche sowie professionsethische Positionierung andererseits.

Die Erkenntnisse aus der Forschungsarbeit zeigen, dass die Sozialarbeitenden in „Zweifelsfällen“ Strategien entwickeln, um trotz Unsicherheit Urteilsvermögen herzustellen und - im Bewusstsein, dass ihnen als Entscheider_innen eine Schlüsselrolle zukommt - um ihr fachliches Mandat so zu definieren, dass es für sie akzeptierbar und mit ihren fachlichen und ethischen Grundprinzipien vereinbar ist. Eine dieser Strategien besteht darin, die Meinungen und Auskünfte Dritter hinzuzuziehen. So werden beispielsweise die betreuenden Fachkräfte in der Jugendhilfeeinrichtung, in der die jungen Geflüchteten im Vorfeld der qualifizierten Inaugenscheinnahme häufig bereits über mehrere Tage gelebt haben, nach ihrer Einschätzung zum Alter befragt. Die situativen Erweiterungen des Teams (von vier zu sechs Augen) eröffnet in diesen Fällen komplementäre Perspektiven zu der Alterseinschätzung. In anderen Fällen werden auch die sprachmittelnden Personen, die ihrerseits häufig aus ähnlichen Kulturkreisen kommen wie die Geflüchteten, in die Konsultationen einbezogen und nach ihrer Meinung zu jugendtypischen Verhaltensweisen, kulturellen Erscheinungsmerkmalen und dem Alter befragt. In diesen Situationen nehmen sie verschiedene Rollen ein - als Kulturmittelnde, Länderexperten und -expertinnen, Mitentscheidende -, wodurch sie teilweise de facto in die Entscheidungsfindung eingebunden werden. Der dadurch vorgenommene Rollenwechsel soll zwar als Ergänzung und Rückkopplung zur Urteilsfindung beitragen, birgt jedoch gleichzeitig das Risiko der Instrumentalisierung dieser Personengruppe.

\section{Schlussfolgerungen und Diskussionsansätze}

Qualifizierte Inaugenscheinnahmen sind aufgrund von Unsicherheit, strategischer Kommunikation sowie vage formulierten, rechtlichen Vorgaben als überkomplex zu bewerten: Von den Sozialarbeitenden werden Einschätzungen erwartet, die mit spezifischen Dilemmata und Fallstricken verbunden sind. Daran anschließend können drei grundsätzliche Diskussionsansätze formuliert werden:

1. Die qualifizierte Inaugenscheinnahme ist ein Mandat der Kinder- und Jugendhilfe und der Sozialen Arbeit. In einigen Bundesländern lässt sich jedoch beobachten, dass sich in der Anwendungspraxis restriktive Haltungen durchsetzen (Karpenstein und Rohleder 2021, S. 26) und qualifizierte Inaugenscheinnahmen u. a. die Form von Glaubwürdigkeitsprüfungen annehmen, die an nicht jugend- 
gerechte Asylanhörungen erinnern (z. B. Millbank 2009; Jubany 2011; Dahlvik 2018). Der Einzug ordnungspolitischer Logiken in die Praxis der qualifizierten Inaugenscheinnahme ist Ausdruck der spannungsreichen Rechtsstellung der Kategorie „UMA“ zwischen Kinder- und Jugendhilfe- und Ausländerrecht und stellt die Frage nach der Positionierung und dem fachlichen Selbstverständnis der Sozialen Arbeit.

2. Die qualifizierte Inaugenscheinnahme steht zunehmend in „Konkurrenz“ zu anderen Verfahren und Behörden, für die die Altersentscheidung des Jugendamts keine Bindungswirkung hat. Dadurch kommt es zu Kompetenz- und Machtverschiebungen. Außerdem werden verstärkt Ausländerbehörden und Mediziner_innen an der Alterseinschätzung beteiligt, was zu Verunsicherung führt (Karpenstein und Rohleder 2021, S. 26). Diese Entwicklung stellt die interprofessionelle Zusammenarbeit zur Diskussion. Zudem stellt sie die Frage nach möglichen und in Fällen der Unsicherheit fachlich gebotenen Sequenzierungen der Alterseinschätzung: Eine wiederholte (zweite, dritte) Inaugenscheinnahme junger Geflüchteter durch Sozialarbeitende und/ oder psychologische Fachkräfte, wie sie in Deutschland in den Handlungsempfehlungen der Bundesarbeitsgemeinschaft der Landesjugendämter (2017, S. 57) sowie im nationalen Jugendhilferecht in anderen Ländern, wie z. B. in Frankreich, explizit vorgesehen sind, wird derzeit durch kurze Fristen im Rahmen der qualifizierten Inaugenscheinnahme erschwert, bzw. verunmöglicht.

3. Darüber hinaus zeigt dieser Beitrag, dass die Kopplung von Altersgrenzen an Entwicklungsstufen, äußeres Erscheinungsbild und mündliche Aussagen gerade im Kontext von Flucht wenig plausibel ist. Die Ermittlung einer rechtlichen Altersgrenze (18 Jahre), die de facto über Inklusion und Exklusion, Regularität und Irregularität, Schutz und mögliche Abschiebung entscheidet, lenkt den Blick darüber hinaus auf das prinzipielle Verhältnis zwischen allgemein gefassten Alterskategorien, aus denen sich ein rechtlich gefasster und universell in der UNKinderrechtskonvention begründeter Hilfebedarf ableitet, und individuellen Hilfebedarfen, die auch über 18 Jahre hinaus noch fortbestehen können.

Aus diesen Diskussionsansätzen ergibt sich abschließend folgendes Bild: Alter ist ein Politikum und die Urteilsbildung im Rahmen der qualifizierten Inaugenscheinnahme ist verschiedenen Einflussfaktoren ausgesetzt. In diesem Spannungsfeld ist die Soziale Arbeit dazu aufgefordert, sich gegenüber anderen Professionen und Behörden (Kompetenz- und Machtverschiebungen) sowie ihrem eigenen Mandat (Übernahme einer Entscheiderrolle) zu positionieren.

\section{ส้อ}

Eingegangen. 14. April 2021

Angenommen. 11. Mai 2021

Funding. Open Access funding enabled and organized by Projekt DEAL.

Open Access. Dieser Artikel wird unter der Creative Commons Namensnennung 4.0 International Lizenz veröffentlicht, welche die Nutzung, Vervielfältigung, Bearbeitung, Verbreitung und Wiedergabe in jeglichem Medium und Format erlaubt, sofern Sie den/die ursprünglichen Autor(en) und die Quelle ordnungsgemäß nennen, einen Link zur Creative Commons Lizenz beifügen und angeben, ob Änderungen vorgenommen wurden.

Die in diesem Artikel enthaltenen Bilder und sonstiges Drittmaterial unterliegen ebenfalls der genannten Creative Commons Lizenz, sofern sich aus der Abbildungslegende nichts anderes ergibt. Sofern das betreffende Material nicht unter der genannten Creative Commons Lizenz steht und die betreffende Handlung nicht nach gesetzlichen Vorschriften erlaubt ist, ist für die oben aufgeführten Weiterverwendungen des Materials die Einwilligung des jeweiligen Rechteinhabers einzuholen.

Weitere Details zur Lizenz entnehmen Sie bitte der Lizenzinformation auf http://creativecommons.org/licenses/by/4.0/deed.de.

1. Die Bezeichnung „unbegleitete ausländische Kinder und Jugendliche" entspricht der rechtlichen Terminologie nach \$42 SGB VIII. In der Fachdiskussion der Sozialen Arbeit wird auch von ,unbegleiteten minderjährigen Flüchtlingen" (UMF) gesprochen. In diesem Artikel wird der Ausdruck „junge Geflüchtete“ verwendet. Er umfasst all diejenigen jungen Menschen mit Fluchterfahrung, deren Alter noch nicht festgelegt wurde und schließt auch jene mit ein, die keinen Asylantrag stellen und die nicht als Flüchtlinge anerkannt sind.

2. Diese gegenläufige Entwicklung folgt dem allgemeinen Trend der Fluchtmigration nach Deutschland bzw. Frankreich. Gründe hierfür liegen in der Verschiebung der Fluchtrouten vom östlichen zum westlichen Mittelmeer, in den sozialen Profilen der jungen Geflüchteten (vor allem ehemalige französische Kolonien wie Guinea, Elfenbeinküste und Mali) sowie im Wandel des politischen Diskurses und der Flüchtlingspolitik der beiden Länder.

3. Eine instruktive Darstellung des rechtlichen Rabmens und der fachlichen Standards der Alterseinschätzung findet sich u. a. in Gonzalez Mendez de Vigo und Wiesinger (2019).

\section{Literatur}

Brinks, S., Dittmann, E., \& Müller, H. (2016). Handbuch unbegleitete minderjährige Flüchtlinge. Regensburg: Walhalla.

Bundesarbeitsgemeinschaft Landesjugendämter (2014). Handlungsempfehlungen zum Umgang mit unbegleiteten minderjährigen Flüchtlingen Inobhutnahme, Clearingverfahren und Einleitung von Anschlussmaßnahmen. Saarbrücken. http://www.bagljae.de/downloads/118_handlungsempfehlungen-umf_2014.pdf. Zugegriffen: 15. März 2021. 


\section{Extrablick: Urteilsbildung in der Sozialen Arbeit}

Bundesarbeitsgemeinschaft Landesjugendämter (2017). Handlungsempfehlungen zum Umgang mit unbegleiteten Minderiährigen Verteilungsverfahren, Maßnahmen der Jugendhilfe und Clearingverfahren. 2. Aktualisierte Fassung. Saarbrücken. http://www.bagljae.de/downloads/128 handlungsempfehlungen-zum-umgang-mit-unbge.pdf. Zugegriffen: 15 . März 2021.

Bundesärztekammer (2016). Medizinische Alterseinschätzung bei unbegleiteten jungen Flüchtlingen. Deutsches Ärzteblatt, 10(473), A1-A6. Stellungnahme der Zentralen Ethikkommission bei der Bundesärztekammer.

Bundesgesetzblatt (2015). Teil I Nr. 42, ausgegeben zu Bonn am 30. Oktober 2015. Gesetz zur Verbesserung der Unterbringung, Versorgung und Betreuung ausländischer Kinder und Jugendlicher, vom. 28. Oktober 2015.

Carayon, L., Mattiussi, J., \& Vuattoux, A. (2018). Soyez cohérent, jeune homme! Enjeux et non-dits de l'évaluation de la minorité chez les jeunes étrangers isolés à Paris. Revue française de Science Politique, 68(1), 31-52. https://doi.org/10.3917/rfsp.681.0031.

Carayon, L., Mattiussi, J., \& Vuattoux, A. (2020). Étrange jeunesse, jeunesse étrangère. Formes de culturalisme dans l'évaluation des jeunes isolés étrangers. Agora débats/jeunesses, 84(1), 109-124. https://doi.org/10.3917/ agora.084.0109.

Dahlvik, L. (2018). Inside asylum bureaucracy: organizing refugee status determination in Austria. Cham: Springer.

Destatis (2020). Jugendämter nahmen 2019 rund 49500 Kinder zu ihrem Schutz in Obhut. Pressemitteilung Nr. 363 vom 17. September 2020. https://www.destatis.de/DE/Presse/Pressemitteilungen/2020/09/ PD20_363 225.html. Zugegriffen: 15. März 2021.

Deutsche Bundesregierung (2020). Bericht der Bundesregierung zur Situation unbegleiteter Minderjähriger in Deutschland. Drucksache 19/17810. https://dipbt.bundestag.de/doc/btd/19/178/1917810.pdf. Zugegriffen: 15. März 2021.

Deutscher Caritasverband e. V. (2018). Position des Deutschen Caritasverbandes Gesetzliche Regelung zur Bestimmung des Alters bei unbegleiteten minderjährigen Schutzsuchenden. Bericht in der Reihe „Fluchtpunkte “. https://asyl-net-migration-bergstrasse.de/wp-content/uploads/2016/11/ fluchtpunkte_05-Altersfeststellung.pdf. Zugegriffen: 15. März 2021.

Europarat (2019). We are children, hear us out! Children speak out about age assessment. Report on consultations with unaccompanied children on the topic of age assessment. https://rm.coe.int/we-are-children-hear-us-outchildren-speak-out-about-age-assessment-re/16809486f3. Zugegriffen: 15. März 2021.

European Asylum Support Office (2018). EASO practical guide on age assessment: second edition. Valetta. https://op.europa.eu/en/publicationdetail/-/publication/9039fb04-3c71-11e8-b5fe-01aa75ed71a1/language-en. Zugegriffen: 15. März 2021.

Gonzalez Mendez De Vigo, N., \& Wiesinger, I. (2019). Alterseinschätzung. Rechtlicher Rahmen, fachliche Standards und Hinweise für die Praxis. Bericht erstellt im Rahmen des Projektes „Blick nach vorn“. https://b-umf.de/src/wp-content/uploads/2019/07/2019_07_arbeitshilfe_alterseinschaetzung.pdf. Zugegriffen: 15. März 2021.

Habermas, J. (1982). Handlungsrationalität und gesellschaftliche Rationalisierung (2. Aufl.). Bd. 1. Frankfurt am Main: Suhrkamp.

Jubany, O. (2011). Constructing truths in a culture of disbelief. Understanding asylum screening from within. International Sociology, 26(1), 7494. https://doi.org/10.1177/0268580910380978.

Karpenstein, J., \& von Nordheim, F. (2020). Die Situation unbegleiteter minderjähriger Flüchtlinge in Deutschland. Auswertung der Online-Umfrage 2019. Bericht erstellt im Rahmen des Projektes „Gut Ankommen“. https://b-umf.de/src/wp-content/uploads/2020/01/2019_12_17_bumfumfrage2019_v04.pdf. Zugegriffen: 15. März 2021.

Karpenstein, J., \& Rohleder, D. (2021). Die Situation geflüchteter junger Menschen in Deutschland. Bericht erstellt im Rahmen des Projektes „Gut Ankommen“. https://b-umf.de/src/wp-content/uploads/2021/04/webversion_onlineumfrage2020.pdf. Zugegriffen: 19. Apr. 2021.

Luhmann, N. (2014). Vertrauen: ein Mechanismus der Reduktion sozialer Komplexität (5. Aufl.). Konstanz: UVK. Originalausgabe von 1968

Millbank, J. (2009). The ring of truth: a case study of credibility assessment in particular social group refugee determinations. International Journal of Refugee Law, 21(1), 1-31. https://doi.org/10.1093/ijrl/een040.

Montagut, M. (2016). L'emprise de la torture: les troubles langagiers des demandeurs d'asile face aux attentes institutionnelles. Langage et société, 4(158), 89-105. https://doi.org/10.3917/ls.158.0089.
Parusel, B. (2015). Unbegleitete Minderjährige auf der Flucht. Aus Politik und Zeitgeschichte, 65(25), 31-38.

Scherr, A. (2014). Unbegleitete minderjährige Flüchtlinge - ein Grenzfall. Soziale Passagen, 6, 313-317. https://doi.org/10.1007/s12592-014-0171-9.

Tangermann, J., \& Hoffmeyer-Zlotnik, P. (2018). Unbegleitete Minderjährige in Deutschland. Herausforderungen und Maßnahmen nach der Klärung des aufenthaltsrechtlichen Status. Fokusstudie der deutschen nationalen Kontaktstelle für das Europäische Migrationsnetzwerk (EMN)

UNICEF (2019). Jedes vierte Kind weltweit existiert offiziell nicht. https:// www.unicef.de/informieren/aktuelles/presse/2019/unicef-report-geburtenregistrierung/208002. Zugegriffen: 15. März 2021.

VG Hannover (2016). Unzureichende Alterseinschätzung vor Beendigung der Inobhutnahme eines UMF. Beschluss v. 11.11.2016-3 B 5176/16

VG München (2016). Vorläufige Inobhutnabme eines unbegleiteten minderiährigen Flüchtlings. Beschluss v. 07.12.2016-M 18 K 16.4361

VGH München (2016). Streit über Verpflichtung zur vorläufigen Inobhutnahme eines unbegleiteten minderjährigen Flüchtlings. Beschluss $v$. 18.08.2016-12 CE 16.1570

VGH München (2017). Feststellung der Minderjährigkeit bedarf einer qualifizierten Inaugenscheinnabme. Beschluss v. 05.04.2017-12 BV 17.185 